\title{
EDITORIAL
}

\section{Pattern of Research Trend Emerging from Small Data}

\author{
Ji-Dong Gu \\ Environmental Engineering, Guangdong Technion Israel Institute of Technology, 241 Daxue Road, Shantou, Guangdong 515063, China
}

\begin{abstract}
Identification and prediction of the current ongoing and future research trends are critically important to research scientists to be on track of the significantly important topics and also ahead of the others if all possible. Such information can be extrapolated by mining the existing data available from different databases to delineate the important research topics that many are working on and also the emerging ones that attract attention. Because of the readily availability of online published articles in Open Access mode and instant information in real time on viewing number, reads and citations, a simple summary of the papers published in this journal over the past 4 years indicated clearly the most viewed research articles and topics are in line with the main stream information available, namely novel dehalogenase, thermophilic organisms and biotechnological application in bioleaching, souring inhibition in oil reservoirs, and the current public interest on plastics. This information can be used in refining one's specific research to target for popularity and visibility.
\end{abstract}

Keywords: Dehalogenase, thermophilic bioleaching, oilfield souring, plastics

Correspondence to: Ji-Dong Gu, Environmental Engineering, Guangdong Technion Israel Institute of Technology, 241 Daxue Road, Shantou, Guangdong 515063, China; E-mail: jidong.gu@gtiit.edu.cn

Received: November 5, 2020; Accepted: December 10, 2020; Published Online: December 30, 2020.

Citation: Ji-Dong Gu, 2020. Pattern of Research Trend Emerging from Small Data. Applied Environmental Biotechnology, vol. 5(2): 1-2. http://doi.org/10.26789/AEB.2020.02.001

Copyright: Pattern of Research Trend Emerging from Small Data. @ 2020 Ji-Dong Gu. This is an Open Access article published by Urban Development Scientific Publishing Company. It is distributed under the terms of the Creative Commons Attribution-Noncommercial 4.0 International License, permitting all non-commercial use, distribution, and reproduction in any medium, provided the original work is properly cited and acknowledged.

Academic publishing is becoming more increasingly competitive for both authors and publishers on both sides of this business. Researchers as authors are required not only to publish to avoid perish, but also to bring high visibility and citations to stay in the research arena. To have a successful career, the initial and most important step is to identify the most promising and important research topics based on knowledge and also the published articles available, so that the results can be attractive to publisher who screen and select the hottest research papers possible to gain wide publicity and the dominance of the publishing market. Delineation of the published information is an essential part of the research activities engaged by active scientists to carry out data mining from articles available for an accurate identification of the current research trend and the future direction. Bibliometrics is a new field developed for this purpose. Most of the analysis and reports are mainly made based on the available database, Web of Science as the main source of such data, for further analysis with more defined question or scope of the data acquisition for further processing and analysis. Such exercise provides invaluable information for researchers to learn the results of such analysis and evaluate the results in many ways to use the meaningful information for planning the on-going and future research accordingly.

Applied Environmental Biotechnology (AEB) is a relatively 'new kid on the block' with a short history of publication, starting for the inaugural issue in 2016, and then 2 issues in the subsequent years from 2017 to 2019 . It is expected that 2 issues will be published in 2020. With such a short period of publication and also a small number of published papers under this journal title, less than 60 articles in total, the viewing statistics by the web clearly indicates that 4 of them have attracted the highest attention to the global readers before the journal has been indexed in SCI database.

Further examination of the published articles in this journal indicated that the topics of interest to readers the most by this journal are on enzymes, the protein structure of the metal center of a dehalogenase hydrolyzing chlorothalonil (Chen et al., 2010) and a low-temperature endo- $\beta$-1,4-mannase from Bacillus subtilis TD7 with expression in E. coli (Li et al., 2018); the evolutionary relationship between ammoniaoxidizing archaea (AOA) and ammonia-oxidizing bacteria (AOB) (Liu et al., 2017); and the current plastic issue to our society $(\mathrm{Gu}, 2017)$. The immediate following these with the highest attention include inhibition of sulfate-reducing activity in oilfield (Liu et al., 2016) and bioleaching (Maulani et al., 2016). In addition, several papers on waste water treatment and anammox process have received attention for a close to a thousand viewing.

The scope of this journal is to publish manuscripts that are relevant to the environment and biotechnology of the different ecosystems and environments in a very broad sense. Since the journal is still at a stage prior to receiving the SCI indexing and also the impact factor (IF), submissions to this journal are not driven by the outcome recognition at this moment, probably faith or investment. However, the attractions to the articles published in this journal are without doubt by the titles and the contents of them plus the Open Access of the articles in full text publicly, which are in line with the promotion of public accessibility of the scientific information globally. Using this as a guide for the future, it can be said as a summary on the increasing attention on any articles 
to be published in the followings: 1) that newly discovered microorganisms and their biochemical capabilities; 2) fundamental process and mechanisms; and 3) publicized issues by mass media and scientific community.

\section{Conflict of Interest}

Author declares that there is no conflict of interest in this research.

\section{Ethical approval}

This article does not contain any studies with human participants or animals performed by the author involved.

\section{References}

Chen H, Wang H, Wang T, Huang S, Zang X, Li S, Jiang J, 2016. Identification of the metal center of chlorothalonil hydrolytic dehalogenase and enhancement of catalytic efficiency by directed evolution. Applied Environmental Biotechnology, 1(1): 30-37.

http://doi.org/10.26789/AEB.2016.01.003
Gu J.-D., 2017. Biodegradability of plastics: the pitfalls. Applied Environmental Biotechnology, 2(1): 59-61. http://doi.org/10.26789/AEB.2017.01.008

Liu S, Hu J-J, Shen J-K, Chen S, Tian G-M, Zheng P, Lou L-P, Ma F, Hu B-L, 2017. Potential correlated environmental factors leading to the niche segregation of ammonia-oxidizing archaea and ammonia-oxidizing bacteria: A review. Applied Environmental Biotechnology, 2(1): 11-19.

http://doi.org/10.26789/AEB.2017.01.002

Li, C-y, Liu F-F, Ye, J, Liu J-F, Yang S-Z, Zhang H-Z, Mu B-Z, 2018. A low-temperature active endo- $\beta$-1,4-mannanase from Bacillus subtilis TD7 and its gene expression in Escherichia coli. Applied Environmental Biotechnology, 3(2): 17-25. http://doi.org/10.26789/AEB.2018.02.004

Liu J-F, Wu W-L, Yao F, Wang B, Zhang B-L, Mbadinga SM, Gu J-D, Mu B-Z, 2016. A thermophilic nitrate-reducing bacterium isolated from production water of a high temperature oil reservoir and its inhibition on sulfate-reducing bacteria. Applied Environmental Biotechnology, 1(2): 35-42. http://doi.org/10.26789/AEB.2016.02.004

Maulani N, Li Q, Sand W, Vera M, Zhang R, 2016. Interactions of the extremely acidophilic archaeon Ferroplasma acidiphilum with acidophilic bacteria during pyrite bioleaching. Applied Environmental Biotechnology, 1(2): 43-55. http://doi.org/10.26789/AEB.2016.02.006 